\title{
Der Philosoph als Briefschreiber
}

\section{Zur Bedeutung der literarischen Form von Senecas Briefen an Lucilius}

Die Briefe an Lucilius ${ }^{1}$ wurden zwar als bedeutendstes Werk Senecas bezeichnet ${ }^{2}$, sie wurden aber auch scharf kritisiert. Glaubt man den Kritikern, so tragen sie schlecht geordnete Überlegungen vor, reihen einzelne Einfälle aneinander und entwickeln keine kohärente Philosophie. Immer wieder wurde Seneca vorgeworfen, er kombiniere eklektizistisch unterschiedliche Positionen und seine Überlegungen seien oberflächlich. Das nicht-systematische Vorgehen in den Briefen an Lucilius wurde als eindeutiges Defizit bewertet. ${ }^{3}$

Im folgenden möchte ich zeigen, daß eine solche Beurteilung nicht gerechtfertigt ist. Eine Untersuchung der Philosophiekonzeption Senecas macht deutlich, daß die Briefform keine bloß äußerliche Einkleidung der vorgetragenen Überlegungen ist.

\section{I.}

Bei den 124 Briefen Senecas an Lucilius handelt es sich um eine Briefsammlung, die nur die Schreiben eines der beiden Beteiligten mitteilt. Durch wiederholte Anreden, Rekapitulationen von Äußerungen des Lucilius in Senecas Briefen und durch Bezugnahmen auf die jeweiligen Lebensumstände des Angeredeten wirken die Texte mitunter wie authentische Stücke einer Privatkorrespondenz.

1 L. Annaeus Seneca, Ad Lucilium epistulae morales - An Lucilius Briefe über Ethik, ed. M. Rosenbach, Bde. I-II, Darmstadt 1980-1984.

2 "[...] les Epistres de Seneque, qui est la plus belle partie des ses escrits, et la plus profitable." M. de Montaigne, CEuvres complètes (Bibliothèque de la Pléiade), Paris 1962, S. 392. Vgl. auch A.A. Long, Hellenistic Philosophy - Stoics, Epicureans, Sceptics, London 1986, S. 233; D. A. Russell, Letters to Lucilius, in: C.D. N. Costa (ed.), Seneca, London/Boston 1974, S. 79; I. Hadot, Seneca und die griechisch-römische Tradition der Seelenleitung, Berlin 1969, S. 125 und R.M. Gummere, Seneca the Philosopher and his Modern Message, New York 1963, S. 51.

3 "[...] il est impossible de voir dans cette collection de lettres un plan méthodique d'ensemble." E. Albertini, La Composition dans les ouvrages philosophiques de Sénèque, Paris 1923, S. 132f. Von der philosophischen Oberflächlichkeit Senecas spricht G.M. Ross, Seneca's Philosophical Influence, in: Costa, Seneca, S. 117. Beschränkte philosophische Fähigkeiten werden Seneca von 1.G. Kidd attestiert. Vgl. ders., Moral Actions and Rules in Stoic Ethics, in: J. M. Rist (ed.), The Stoics, Berkeley/Los Angeles/London 1978, S. 251. 
Wie aus einem Hinweis Senecas hervorgeht, handelt es sich aber nicht um rein private Briefe, die uns nur durch einen Zufall überliefert wurden, sondern um ein zur Veröffentlichung bestimmtes Werk. ${ }^{+}$

Den thematischen Schwerpunkt dieses Werks bilden ethische Fragen. Seneca will keine eigenständige Ethik als theoretische Teildisziplin neben Logik und Physik ${ }^{5}$ erarbeiten. Theoretische Probleme, etwa die Definition ethischer Grundbegriffe, stehen nicht im Vordergrund, stattdessen sollen die Briefe dem Adressaten helfen, sein Leben vernünftig zu gestalten. Sie tun dies nicht nur, indem Rat erteilt, Empfehlungen ausgesprochen und Vorschriften formuliert werden. Sie vergegenwärtigen das Bemühen um eine vernunftgemäße Lebensweise, indem der Absender sich selbst Rechenschaft über seine Lebensführung ablegt: Seneca spricht nicht nur in der Rolle des Lehrers, er nimmt selbst die Rolle eines Lernenden ein. ${ }^{6}$

Die Konzentration auf Fragen der Lebenspraxis impliziert nun keineswegs eine strikte Abwendung von der philosophischen Ethik als Wissensbildung in Theorieform. Seneca berücksichtigt durchaus die von verschiedenen Schulen formulierten Positionen und steht auf der Grundlage der stoischen Philosophie. Insbesondere im Mittel- und Schlußteil der Briefsammlung gewinnen teilweise detaillierte Auseinandersetzungen mit einzelnen philosophischen Lehrsätzen zunehmend an Gewicht. Solche theoretischen Erörterungen und Diskussionen bleiben aber stets an das grundlegende Interesse zurückgebunden, dem Leser Wege aufzuzeigen, wie er sein Leben vernünftig gestalten kann. Eine gewisse Skepsis bezüglich der Erfolgsaussichten dieses Programms wird in der folgenden Äußerung deutlich:

"Worte bestätige durch Tatsachen. Eine andere Absicht haben Menschen, die Vorträge halten und nach dem Beifall der Zuhörer haschen; eine andere haben die, die junger und müßiger Menschen Ohren durch vielfältige und gewandte Erörterungen fesseln: handeln lehrt die Philosophie, nicht reden, darauf dringt sie, daß nach seinem eigenen Gesetz ein jeder lebe, damit nicht zur Rede das Leben in Widerspruch stehe oder in sich selbst (widersprüchlich sei), daß es bei allen seinen Handlungen nur eine Färbung gebe. Das ist die wichtigste Aufgabe der Weisheit und der Beweis für sie, daß mit den Worten die Taten übereinstimmen, daß man selber in jeder Situation sich gleich und derselbe ist. ,Wer wird das leisten? - Wenige, manche dennoch. $\aleph^{7}$

4 Ep. $21,5$.

5 Die Stoa gliederte die Philosophie üblicherweise in die drei Bereiche Logik, Physik und Ethik. Vgl. K. Hülser (ed.), Die Fragmente zur Dialektik der Stoiker, Bd. I, Stuttgart-Bad Cannstatt 1987, S. 2-5. Einen instruktiven Überblick über die stoische Philosophie gibt das erste Kapitel ("Stoicism in Antiquity") in: M.L. Colish, The Stoic Tradition from Antiquity to the Early Middle Ages, Bd. I: Stoicism in Classical Latin Literature, Leiden 1985, S. 7-60.

6 Vgl. Epp. 27,1; 34,2; 87,4f.

7 Ep. 20,1 f.: " [...] verba rebus proba. Aliud propositum est declamantibus et adsensionem coronae captantibus; aliud his, qui iuvenum et otiosorum aures disputatione varia aut volubili detinent: facere docet philosophia, non dicere, et hoc exigit, ut ad legem suam quisque vivat, ne orationi vita dissentiat aut ipsa inter se vita, cut) unus sit omnium actionum color [sit]. Maximum hoc est et officium sapientiae et indicium, ut verbis opera concordent, ut ipse ubique par sibi idemque sit. Quis hoc praestabit? Pauci, aliqui tamen.* 
Senecas Briefe präsentieren die Philosophie primär als eine ars vitae, als "Technik" der Lebensführung. ${ }^{8} \mathrm{Da}$ die Formulierung eines Begriffs vernünftiger Lebensführung nicht bewirkt, daß die Menschen ihr Handeln nach Maßgabe dieses Begriffs bestimmen, sieht sich Seneca dazu veranlaßt, als Philosoph Strategien zu erproben, die die Umsetzung der im Rahmen der philosophischen Ethik gewonnenen Einsichten in den Lebensvollzug der Subjekte ermöglichen. Der zentrale Gegenstand Senecas ist das Problem, wie man von einem ethischen Wissen ausgehend ein bestimmtes Können - die Fähigkeit, dem ethischen Wissen entsprechend zu handeln - erwirbt.

Dabei ist er von der Vorstellung geleitet, daß das Ziel einer Übereinstimmung von philosophischer Ethik und Lebenspraxis nicht durch einen einmaligen Entschluß, sondern nur durch schrittweise Modifikationen der Lebensfuhrung erreicht werden kann.

Die Philosophie als ars vitae unterstützt die Einübung neuer, ethisch gerechtfertigter Verhaltensweisen und Einstellungen:

"Alle sind wir vorbelastet: sittliche Haltung zu lernen heißt Fehlhaltung zu verlernen. $"$ "

II.

Um ihre Aufgabe zu erfüllen, hält die Philosophie ein Repertoire an Übungen bereit, die der Philosophierende täglich ausfuhren soll. ${ }^{10}$ Das ethische Trainingsprogramm setzt sich aus den folgenden Elementen zusammen: Lektüre, Memorieren, schriftliches Durcharbeiten, Gespräch, Vergegenwärtigung der Exempla, Selbstprüfung, Meditation.

Im folgenden sollen diese Tätigkeiten kurz erläutert werden:

8 Diesem Aspekt des Philosophiebegriffs Senecas hat I. Hadot eine ausführliche Untersuchung gewidmet: Hadot, Seneca. Diese Arbeit weist schon in die hier verfolgte Richtung, wenn konstatiert wird, daß eine adäquate Beurteilung der Texte Senecas nur möglich ist, "[...] wenn man ihre Form von ihrer Funktion her zu verstehen sich bemüht." Ebd., S. 38.

9 Ep. 50,7: "Omnes praeoccupati sumus: virtutes discere vitia dediscere (est)." Rosenbachs Übersetzung von `vitium` durch `Fehlhaltung` scheint mir außerordentlich glücklich, da sie eine Annäherung der Ausfuhrungen Senecas an christliche Vorstellungen vermeidet.

10 In umfassender Weise hat P. Hadot den Übungscharakter der Philosophie untersucht. Vgl. die Aufsatzsammlung P. Hadot, Exercices spirituels et philosophie antique, Paris 1987.

In seiner Abhandlung "Exercices spirituels» (S. 15-58) kommt Hadot zu dem Ergebnis, daß die antike Philosophie insgesamt unter dem Aspekt der Einübung in eine Lebensform zu begreifen ist: "Il n'est donc pas possible de comprendre les théories philosophiques de l'Antiquité sans tenir compte de cette perspective concrète qui leur donne leur véritable signification." (S. 51) "La philosophie apparait alors, dans son aspect originel, non plus comme une construction théorique, mais comme une méthode de formation à une nouvelle manière de vivre [... ]" (S. 56).

In bezug auf die spätantike Philosophie greift $\mathrm{M}$. Foucault diese Konzeption auf und analysiert sie als eine "culture de soi«. M. Foucault, La culture de soi, in: Le souci de soi (Histoire de la sexualité, Bd. III), Paris 1984, S. 51-85. In Foucaults Ausfuhrungen wird der Rigorismus der Ethik bei Seneca zugunsten einer Ästhetisierung der Lebensführung abgeschwächt. Vgl. zu dieser Arbeit des späten Foucault die differenzierenden Bemerkungen Hadots "Un dialogue interrompu avec Michel Foucault. Convergences et divergences" (S. 229-233). 


\section{Lektüre}

Die Lektüre verfolgt nicht den Zweck, ein möglichst umfassendes Wissen über einen Gegenstandsbereich zu erschließen, sondern Ansichten anerkannter Autoren zu vermitteln. Das Lesen dient nicht der Zerstreuung, sondern der Konzentration und der Reflexion ethischer Probleme. Seneca warnt ausdrücklich vor einer Überfülle an Lesestoff. ${ }^{11}$ Die tägliche Lektüre soll einen Beitrag zur Einübung vernünftiger Einstellungen leisten. Sie tut dies dadurch, daß sie unbegründete aber fest verwurzelte Vorstellungen korrigiert. Standardbeispiele des Stoikers Seneca für solche irrtümlichen Vorstellungen sind die Überbewertung von Reichtum und Macht oder die Furcht vor dem Tod.

In seinem zweiten Brief an Lucilius empfiehlt Seneca die Lektüre mit folgenden Worten:

"Es zerstreut der Bücher Menge: daher - weil du nicht lesen kannst, wieviel du (an Büchern) besitzen könntest - ist es genug, zu besitzen, was du lesen kannst.

'Aber baldı, sagst du, ‘will ich dieses Buch aufschlagen, bald jenes.r - Eines verwöhnten Magens Art ist es, vieles zu kosten; sobald es vielfältig und verschieden ist, verunreinigt, nicht nährt es. Anerkannte (Autoren) lies daher stets [... . Etwas Hilfe täglich gegen die Armut, etwas Hilfe gegen den Tod bereite und ebenso gegen sonstiges Unheil $[\ldots]^{12}$

\section{Memorieren}

An die Lektüre schließt sich das Memorieren von einzelnen Aussagen und Maximen an. Im Anschluß an die oben zitierte Passage schreibt Seneca:

"[...] nimm eines heraus, das du an jenem Tag ganz dir zu eigen machen willst. Das tue auch ich selber; aus vielem, was ich gelesen habe, mache ich mir etwas zu eigen. Heute ist es das, was ich bei Epikur gefunden habe $[\ldots]]^{13}$

Aus dem ausgewählten Lesestoff wird beispielsweise eine Sentenz herausgenommen und durchgearbeitet. Hierbei geht es nicht um eine bloße Speicherung des Textmaterials im Gedächtnis, sondern um die Aneignung des Gelesenen. Die Briefe Senecas wenden diese Technik des Einprägens und Durchreflektierens insofern an, als an ihrem Ende meistens ein Diktum mitgeteilt wird, das sich der Adressat einprägen soll.

Im 33. Brief gibt Seneca dieses Verfahren auf. Er legt dar, daß Lucilius nun über ein

11 Ep. 45,1: "[...] lectio certa prodest, varia delectat. Qui, quo destinavit, pervenire vult, unam sequatur viam, non per multas vagetur $[\ldots] "-n[\ldots]$ eine zielbewußte Lektüre nützt, abwechslungsreiche unterhält. Wer zu seinem Ziel gelangen will, verfolge einen einzigen Weg, nicht schweife er über viele $[\ldots]$.

12 Ep. 2,3f.: „Distringit librorum multitudo: itaque cum legere non possis, quantum habueris, satis est habere, quantum legas. 'Sed modo, inquis, hunc librum evolvere volo, modo illum.r Fastidientis stomachi est multa degustare; quae ubi varia sunt et diversa, inquinant, non alunt. Probatos itaque semper lege $[\ldots]$ Aliquid cotidie adversus paupertatem, aliquid adversus mortem auxili compara, nec minus adversus cetera pestes [...]".

13 Ep. 2,4f.:" [...] unum excerpe, quod illo die concoquas. Hoc ipse quoque facio; ex pluribus, quae legi, aliquid adprehendo. Hodiernum hoc est, quod apud Epicurus nactus sum [...]". 
Stadium hinausgelangt sei, in dem die Beschäftigung mit isolierten Zitaten sinnvoll erscheine. Lucilius solle sich nun stoisches Gedankengut nicht in Form einzelner Bruchstücke, sondern in größeren Zusammenhängen aneignen, und er solle selbständig denken. ${ }^{14}$ Das Memorieren erscheint als eine Übung für den Anfänger.

\section{Schriftliche Darlegung}

Während das Memorieren der Einprägung und Aneignung einzelner Einsichten dient, hat die schriftliche Darlegung die Aufgabe, das Gelesene mit dem eigenen Wissen zu vermitteln. Es handelt sich um eine zusammenfassende Aufarbeitung des Lesestoffs, die das neue in das bereits gewonnene Wissen integriert.

Lesen und Schreiben müssen in einem ausgewogenen Verhältnis zueinander stehen:

" [...] die Schriftstellerei (soll), was immer man bei der Lektüre zusammengetragen hat, in ein Ganzes einbringen [...] ,Verdauen` wir es (das Gelesene): sonst geht es nur in unser Gedächtnis über, nicht in unser Wesen. «15

\section{Gespräch}

Das Gespräch steht ebensowenig wie die Lektüre im Zeichen der Zerstreuung und Unterhaltung, sondern dient der gemeinsamen Wissensbildung. Themen solcher problembezogener Gespräche im Freundeskreis sind beispielsweise die Platonische Philosophie (Ep. 58,1), die Begriffe Ursache und Materie (Ep. 65,2) oder die stoische Güterlehre (Ep. 66,5). Seneca gibt in seiner Briefsammlung nicht nur die Themen solcher Gespräche an, er referiert ihren Verlauf und gestaltet längere Abschnitte seiner Briefe als Dialoge mit Lucilius.

Die Briefe ähneln dem Gespräch aber nicht nur durch das Abwägen unterschiedlicher Positionen in Rede und Gegenrede. Seneca betont eine grundsätzliche Ähnlichkeit des Briefs mit dem mündlichen Gespräch. Der Modus der Schriftlichkeit bewirkt keine Behinderung der Verständigung. Der Brief wird als Äquivalent zur mündlichen Unterredung dargestellt. ${ }^{16}$

14 Ep. 33,8: "Meminisse est rem commissam memoriae custodire: at contra scire est sua facere quaeque nec ad exemplar pendere et totiens respicere ad magistrum." -

"Sich zu erinnern bedeutet, einen dem Gedächtnis anvertrauten Sachverhalt zu bewahren: aber hingegen wissen heißt, alles sich zu eigen machen, nicht von einem Vorbild abhängig sein und so oft sich umzusehen nach dem Lehrer."

15 Ep. 84,2.7 (Ergänzungen in Klammern von mir): »[. . .] ut quicquid lectione collectum est, stilus redigat in corpus [...] Concoquamus illa: alioquin in memoriam ibunt, non in ingenium."

16 Ep. 40,1: "Numquam epistulam tuam accipio, ut non protinus una simus." - "Niemals empfange ich einen Brief von dir, ohne daß wir nicht sofort zusammen sind." Vgl. in diesem Zusammenhang auch Epp. 38,1; 67,2; 75,1. 


\section{Vergegenwärtigung der Exempla}

Ein weiteres konstitutives Element der Philosophie als ars vitae bei Seneca besteht in der Veranschaulichung angestrebter Verhaltensweisen und Einstellungen durch bestimmte Personen. Zu den von Seneca am häufigsten angefuhrten Leitbildern gehören Sokrates und Cato. ${ }^{17}$ Diesen Vorbildern wird eine wichtige erzieherische Funktion zugeschrieben: der einzelne korrigiert sein Verhalten, indem er es nach dem Muster der exemplarischen Persönlichkeit ausrichtet:

"Jemanden habe die Seele, den sie scheue, aufgrund dessen moralischen Gewichtes sie auch ihr Innerstes unschuldiger mache. Glücklich jener, der nicht durch seine Gegenwart nur, sondern auch wenn man an ihn denkt, besser macht [...] Wähl den, bei dem dir gefällt Lebensform und Art zu sprechen und das Gesicht selbst, die Gesinnnung in sich zeigend: ihn halte dir stets vor Augen als Wächter oder als Beispiel."18

Die Briefe Senecas sind voll von Darstellungen vorbildhaften Verhaltens. Eine Vielzahl narrativer Skizzen stellt dem Leser den Zielzustand der ethischen Bemühungen in anschaulicher Form vor Augen. Wie für die übrigen Techniken gilt auch hier, daß es wichtig ist, sie nicht sporadisch sondern beständig anzuwenden und zu üben.

\section{Selbstprüfung}

Das Vorhaben, die eigene Lebensführung nach ethischen Prinzipien auszurichten, führt zu einer Form der Selbstkontrolle, die Klarheit darüber verschaffen soll, inwieweit Annäherungen an das gesetzte Ziel erfolgt sind. Diese Selbstprüfung hat den Charakter einer strengen Befragung:

"[...] soweit du kannst, mach dir Vorwürfe, verhöre dich; des Anklägers Aufgabe nimm zuerst wahr, dann des Richters, zuletzt des Fürsprechers. Gelegentlich sei schonungslos mit dir. ${ }^{19}$

Diese Überprüfung des eigenen Verhaltens mißt beständig den Abstand zwischen dem angestrebten Ziel und den tatsächlichen Verhältnissen aus. Als Abschluß des Tageslaufs eingesetzt stellt diese Übung eine Abrechnung mit den Taten des Tages dar. ${ }^{20}$ In einem seiner Traktate gibt Seneca die ausführlichste Darstellung dieses Verfahrens:

17 Vgl. beispielsweise Ep. 104, 27-29.

18 Ep. 11,9f.: "Aliquem habeat animus, quem vereatur, cuius auctoritate etiam secretum suum sanctius faciat.

$\mathrm{O}$ felicem illum, qui non praesens tantum, sed etiam cogitatus emendat! [...] Elige eum, cuius tibi placuit et vita et oratio et ipse animum ante se ferens vultus: illum tibi semper ostende vel custodem vel exemplum."

19 Ep. 28,10: "Ideo quantum potes, te ipse coargue, inquire in te; accusatoris primum partibus fungere, deinde iudicis, novissime deprecatoris. Aliquando te offende."

Vgl. auch Epp. 83,2; 101,7.

20 I. Hadot verweist auf den Epikureismus als Herkunftsbereich dieser Übung (Hadot, Seneca, S. 66-71). M. Foucault macht darauf aufmerksam, daß es sich bei dem mexamen de conscience" um ein Element des pythagoreischen Unterrichts handelt (Foucault, Souci de soi, S. 77). 
"Gibt es etwas Schöneres als die Gewohnheit, seinen ganzen Tag so durch und durch zu prüfen? Wie ruhig ist der Schlaf, wie tief und frei, der auf die Selbstmusterung folgt! Täglich verantworte ich mich vor meinem Richterstuhl. Wenn fortgetragen ist das Licht und meine Frau verstummt, die meine Sitte kennt, durchstöbere ich meinen ganzen Tag und nehme meine Taten und Worte wieder vor; gar nichts verhehle ich, nichts übergehe ich. ${ }^{21}$

\section{Meditation}

Die Meditation erscheint bei Seneca in zwei unterschiedlichen Formen als spraemeditatio malorum und als $>$ meditatio mortisı.

Im Fall der spraemeditatio malorum ${ }^{22}$ handelt es sich um die Vorstellung möglicherweise eintretender Unglücksfälle:

"[...] was immer geschehen kann, wollen wir uns als bevorstehend denken. $\ll^{23}$

Die bewußte Lenkung der Vorstellung auf Unglücksfälle wie Mißerfolg, Armut, Krankheit soll das Bewußtsein dafür stärken, daß es sich um unerfreuliche, aber kontingente und insofern gleichgültige Ereignisse handelt. Diese Zustände und Ereignisse entziehen sich zumeist der Verfuigungsgewalt des einzelnen, sie sind dem Subjekt weder als Verfehlungen noch als Leistungen anzurechnen. Da sie die sittliche Substanz der Person nicht direkt berühren, werden sie von den Stoikern als Adiaphora, als moralisch neutral, bezeichnet. ${ }^{24}$

Die antizipierende Meditation soll eine gewohnheitsmäßige Überbewertung solcher Ungliicksfälle abbauen. Ihr Ziel ist eine Gelassenheit, die die Kontingenz der Ereignisse akzeptiert.

Die zweite Form der Meditation ist die smeditatio mortis $<$. Der Gedanke an den Tod spielt zwar bereits im Zusammenhang mit der vorwegnehmenden Vorstellung möglicher Geschehnisse eine Rolle, er wird aber darüberhinaus eigens zum Gegenstand der Reflexion gemacht. In der 'meditatio mortis‘ soll der Gedanke an den Tod einer Schärfung des Bewußtseins der Verantwortlichkeit für das eigene Handeln dienen. Indem täglich die Vorstellung aufgerufen wird, das Ende des Lebens sei gekommen, wird die Aufmerksamkeit von einzelnen Ereignissen im Leben auf das Leben als Ganzes gelenkt. In dieser Form gehört die smeditatio mortis` zu den Verfahren der Selbstprïfung:

21 De ira III, 36, 2 ff. Deutsche Übersetzung zitiert nach I. Hadot, Seneca, S. 69.

"Quicquam ergo pulchrius hac consuetudine excutiendi totum diem? Qualis ille somnus post recognitionem sui sequitur: quam tranquillus, quam altus ac liber, cum aut laudatus est animus aut admonitus et speculator sui censorque secretus cognovit de moribus suis. Utor hac potestate et cotidie apud me causam dico. Cum sublatum e conspectu lumen est et conticuit uxor moris iam mei conscia, totum diem meum scrutor factaque ac dicta mea remetior; nihil mihi ipse abscondo, nihil transeo."

Lateinischer Text nach: L. Annaeus Seneca, Philosophische Schriften, ed. M. Rosenbach, Bd. I, Darmstadt 1969, S. 298.

22 Zur Tradition der spraemeditatio malorum

23 Ep. 24,15: " [...] quicquid fieri potest, quasi futurum cogitemus." Vgl. auch Ep. 91,3ff.

24 Vgl. Ep. 82,10f. 
"Daher ist so $\mathrm{zu}$ ordnen ein jeder Tag, als beschließe er den Zug, vollende und erfülle er das Leben. ${ }^{25}$

Zudem steht der Gedanke an den Tod bei Seneca in enger Verbindung zu den Begriffen der Selbstbestimmung und Freiheit. Wenn in den bisher skizzierten Vorstellungen der Tod als ein von außen auf das Subjekt zukommendes, unhintergehbares Geschehen erscheint, so thematisiert Seneca immer wieder eine weitere Konzeption des Todes, den Freitod. Dieser wird nicht als ein Akt der Verzweiflung, sondern unter bestimmten Bedingungen als eine Handlung der Vernunft charakterisiert:

mDenk an den Tod : wer das sagt, fordert dazu auf, an die Freiheit zu denken. Wer zu sterben gelernt hat, hat verlernt, Sklave zu sein: oberhalb aller Macht steht er, auf jeden Fall außerhalb jeder Macht. Was (können) gegen ihn Kerker, Gefangenschaft und Riegel? Einen freien Ausgang hat er. $\|^{26}$

III.

Die Darstellung des praxisorientierten Aspekts der Philosophie bei Seneca sollte zeigen, daß eine Perspektive unzureichend ist, die den Übungscharakter dieser Philosophie ausklammert. Interpretationen, die die Briefe als Abhandlungen oder Essays lesen, und Untersuchungen, die in doxographischer Absicht die mitgeteilten Lehrsätze registrieren, sind nicht ausreichend. Damit soll keineswegs bestritten werden, daß sich solche Interpretationen auf Senecas Briefsammlung berufen können, da mehrere Briefe tatsächlich als Abhandlungen mit ausführlichen Argumentationsketten angelegt sind.$^{27}$ Die doxographische Perspektive soll also nicht verdrängt, sondern in einen umfassenderen Ansatz integriert werden, der der im Konzept der Philosophie als ars vitae begründeten Anwendungsbezogenheit und dem Einübungscharakter der ethischen Reflexionen gerecht wird. Dabei stellt sich die Frage, aus welchen Gründen gerade die literarische Form der Briefsammlung zu der skizzierten Konzeption der Philosophie paßt und weshalb die Berücksichtigung der literarischen Gestaltung wesentlich ist.

Zunächst kann man hervorheben, daß der Schreiber der Briefe nicht nur einzelne Verfahren einer philosophischen Lebensführung beschreibt, er wendet sie im Schreiben

25 Ep. 12,8: "Itaque sic ordinandus est dies omnis, tamquam cogat agmen et consummet atque expleat vitam."

Vgl. auch Epp. 26,4-8; 49,10; 54,7; 93,6; $101,7$.

26 Ep. 26,10 (Leichte Veränderung der Übersetzung durch mich): " 'Meditare mortem`: qui hoc dicit, meditari libertatem iubet. Qui mori didicit, servire dedidicit: supra omnem potentiam est, certe extra omnem. Quid ad illum carcer et custodia et claustra? Liberum ostium habet."

Vgl. in diesem Zusammenhang W. Kamlah, Meditatio mortis, Stuttgart 1976. Kamlah greift in seiner Argumentation für das Recht auf einen eigenen Tod, die eine Wendung gegen das christliche Todesverständnis impliziert, auf antike Überlegungen zurück. Dabei akzentuiert er ganz im Sinn der stoischen ars vitae die Bedeutung der Einübung angemessener Einstellungen.

27 Vgl. beispielsweise Epp. 94 und 95 mit der Debatte über die Nützlichkeit und Funktion von Vorschriften ('praeceptar) und Lehrsätzen ('decretar). Das Wechselspiel von theoretischer Argumentation und adressatenbezogenem Schreiben in den Lucilius-Briefen wird im einzelnen analysiert von H. Cancik, Untersuchungen zu Senecas Epistulae morales, Hildesheim 1967, S. 13-45. 
selbst an: Er arbeitet seinen Lesestoff auf ${ }^{28}$, er referiert problembezogene Gespräche ${ }^{29}$ und führt den Briefwechsel mitunter wie ein solches Gespräch ${ }^{30}$, er vergegenwärtigt sich exemplarisches Verhalten ${ }^{31}$, er legt Rechenschaft über sein eigenes Verhalten $a b .{ }^{32}$

Der philosophische Brief Senecas zeichnet sich durch den Doppelaspekt von Darlegung eines Programms und exemplarischem Vollzug der Elemente dieses Programms aus. Zwar könnte auch eine systematische oder essayistische Darstellung einzelne Proben der betreffenden Übungen geben; die Briefform ermöglicht aber eine einprägsamere Darstellung des Übungscharakters der Philosophie, als dies in einem Traktat oder Essay möglich wäre. Das hängt hauptsächlich mit der Segmentierung des Gesamttexts in die einzelnen Briefe zusammen, die in nicht genau fixierten zeitlichen Intervallen aufeinander folgen. Um die zeitliche Distanz zwischen den Briefen zu überbrücken, greift Seneca häufig auf bereits Gesagtes zurück. Solche Wiederholungen sind einerseits durch die den Text bestimmende Kommunikationssituation motiviert, andererseits sind sie als Ausdruck des Übungscharakters zu sehen: Nicht das rasche Begreifen der Korrektheit einer syllogistischen Schlußfolgerung stellt Senecas Paradigma philosophischer Arbeit dar, sondern die täglich wiederholten Bemühungen des einzelnen, seinem Leben eine vernünftige Form zu geben. Die häufigen Wiederholungen bestimmter Gedanken demonstrieren diese unablässigen Anstrengungen des Philosophierenden, und der explizite Adressatenbezug trägt dem Umstand Rechnung, daß die philosophische Arbeit auf die Kommunikation mit einem realen oder imaginierten Partner angewiesen ist. Das Werk ist so angelegt, daß sowohl das Schreiben des Autors als auch die Arbeit des Lesers als Übungen im Sinn einer philosophischen ars vitae verstanden werden können.

Die Wahl der Briefform als einer durch den expliziten Bezug des Schreibers auf einen Adressaten definierten Textgattung ist vor allem aber dadurch motiviert, daß die Lösung von Fehlhaltungen und das Einüben neuer Einstellungen einem isolierten Subjekt kaum gelingen kann. Ein in seiner Partikularität befangenes Individuum kann ohne die Öffnung auf ein Gegenüber, das eine Subjektivität überschreitende Rationalität gewährleistet, nicht in den philosophischen Bildungsprozeß eintreten. Der explizite Adressatenbezug des philosophischen Briefs ist daher auch als Ausdruck der Notwendigkeit eines intersubjektiven Austauschs zu sehen. Er ist ein Zeichen für die Angewiesenheit auf den realen oder vorgestellten Anderen, durch den das Philosophieren zu einem therapeutischen Geschehen wird. Als solches wird es durch eine rekurrente Medizin-Metaphorik ${ }^{33}$ ausgewiesen.

28 Epp. 59,7ff.; $87,11-40 ; 94,1 \mathrm{ff}$.

29 Epp. 58; 65,2ff.; 66,5ff.

30 Epp. 14,12ff.; 20,7ff.; 76,1 ff.

31 Epp. 30; 87,9; 120,6f.

32 Ep. 83,1-7.

33 "[...] posterorum negotium ago. Illis aliqua quae possint prodesse conscribo: salutares admonitiones, velut medicamentorum utilium compositiones, litteris mando, esse illas efficaces in meis ulceribus expertus, quae etiam si persanata non sunt, serpere desierunt." - "...] der Nachwelt Angelegenheit betreibe ich. Für sie schreibe ich etwas, was nutzen kann: heilsame Ermahnungen, wie nützlicher Heilmittel Zusammenstellungen, vertraue ich den Buchstaben an; daß sie wirksam sind, habe ich an meinen eigenen Geschwüren erfahren, die, auch wenn sie nicht völlig abgeheilt sind, fortzuwuchern aufgehört haben."

Ep. 8,2; Vgl. auch Epp. 50,4; 52,9; 68,8; 72,6; 75,7; 104,18; $123,17$. 
An dieser Stelle liegt ein Vergleich des philosophischen Briefs mit dem Dialog nahe. Insbesondere der Sokratische Dialog ${ }^{34}$ zeichnet sich durch Eigenschaften aus, die mit den in bezug auf die Lucilius-Briefe getroffenen Feststellungen übereinstimmen. Sowohl der Sokratische Dialog als auch der philosophische Brief Senecas stehen in Opposition zum monologischen Traktat. Beide Gattungen wenden sich gegen ein abgehobenes Systemdenken und beziehen ausdrücklich die Praxis des Philosophierenden in die Erörterungen ein. Für Senecas Briefe sind, ebenso wie für den Dialog Platons, die Bildung des Philosophierenden und die Vermittlung vernünftiger Einstellungen wesentliche Zwecke des Philosophierens. ${ }^{35}$

Unterschiede zwischen den Modellen des Sokratischen Dialogs und des philosophischen Briefs bei Seneca sind aber unübersehbar. Der Sokratische Dialog ist seiner Idee nach durch die aktive Beteiligung mindestens zweier Sprecher geprägt. Dabei unterscheiden sich die Dialogpartner in der Regel dadurch, daß Sokrates den Gang der Unterredung durch sein mäeutisches Können lenkt, während sein Gegenüber die Rolle des Antwortenden übernimmt. Es handelt sich also um eine ungleichgewichtige Beteiligung der Dialogpartner an den gemeinsamen Untersuchungen. Ein wesentliches Ziel des Dialogs ist die Bildung eines autonomen Subjekts. Mittel zur Annäherung an dieses Ziel ist die Unterredung. Im Gegensatz hierzu erreicht das philosophierende Subjekt bei Seneca seine Bestimmung als Vernunftwesen durch eine bewußte Einübung und Eingliederung in eine vorgegebene Lebensordnung.

In beiden Fällen, bei Platon und bei Seneca, läßt sich Philosophie als Lebensform bestimmen, wobei sich die von Seneca modellierte Lebensform von derjenigen Platons durch ihren spezifischen Übungscharakter unterscheidet.

Bei Platons Dialogen handelt es sich nicht um Protokolle realer Gespräche, sondern um literarische Werke. In bezug auf die Lucilius-Briefe kann man überlegen, ob es sich hier ebenfalls gar nicht um die eine Hälfte einer realen Korrespondenz zwischen Seneca und seinem Freund handelt, sondern um ein die Form des Briefs gebrauchendes literarisches Werk. ${ }^{36}$ Im Rahmen einer solchen Hypothese stellt sich allerdings die Frage, weshalb Seneca darauf verzichtet, einen regelrechten Briefwechsel zu komponieren. Aus welchen Gründen greift Seneca zur asymmetrischen Form der Briefsammlung?

Der naheliegendste Grund ist der, daß durch die Aussparung der Antwortbriefe des Lucilius die Adressatenposition nicht vollständig ausgefuillt ist. Dies bedeutet, daß das

34 Vgl.J. Mittelstraß, Versuch über den Sokratischen Dialog, in: ders., Wissenschaft als Lebensform, Frankfurt 1982, S. 138-161.

35 Vgl. Mittelstraß, Versuch, S. 11 und 22.

36 Neben einer Äußerung Senecas, die die Veröffentlichung seiner Briefe thematisiert (Ep. 21,5), spricht ein die Textstruktur betreffender Befund für diese Annahme. Die Briefserie weist eine komplexe Gesamtstruktur auf, in der einzelne Briefe in einer Weise aufeinander zugeordnet sind, wie sie bei einer realen Korrespondenz kaum auftreten kann. Vgl. hierzu H. Cancik, Untersuchungen und G. Maurach, Der Bau von Senecas epistulae morales, Heidelberg 1970. Während die ältere Forschung viel Mühe auf die Suche nach Beweisen für den nicht-fiktionalen Status der Lucilius-Briefe verwendet hat, tritt diese Fragestellung seit Canciks Untersuchung zunehmend in den Hintergrund: "Our appreciation of the work should not be much affected by a failure to resolve to everyone's satisfaction the question whether it in fact is real." D.A.Russell, Letters, S. 76 . 
Werk nach außen hin nicht abgeschlossen ist und der Leser in stärkerer Weise miteinbezogen werden kann, als dies bei einem Briefwechsel der Fall wäre. Über die Instanz des fiktiven Lesers Lucilius wendet sich der Autor an die realen Leser seines Werks. Indem er die Repliken des Lucilius auf seine Ermahnungen, Anregungen und Bitten zu großen Teilen ausspart, läßt er seinen Lesern einen Spielraum, um selbst auf das Gesagte zu reagieren. Die Instanz des Rezipienten ist demnach in die Position des explizit angesprochenen Lucilius und die Position der indirekt angeredeten Leser aufgespalten. Die realen Leser werden als potentielle Teilnehmer an der philosophischen Lebensform miteinbezogen, sie können in die Position des Lucilius einrücken.

Die Instanz des Lucilius ist aber nicht nur für die Seite des Rezipienten der Briefe bedeutsam. Der Schreiber selbst bedarf des Bezugs auf ein Gegenüber, um nicht in ein unangemessenes Monologisieren zu geraten. Da auch der Lehrende noch ein für sich selbst Lernender ist, ist er auf die Vorstellung der Reaktionen des Schülers angewiesen, denn durch diese wirkt das Gelehrte auf den Lehrer zurück. Der imaginierte Dialog Senecas mit dem fiktiven Adressaten Lucilius bindet die Selbstreflexionen des Autors in den umfassenden Zusammenhang der Beförderung einer vernünftigen Lebensführung ein.

Aufgrund der gekennzeichneten Kommunikationsstruktur stellt der philosophische Brief Senecas eine Synthese des Dialogs und der Selbstreflexion dar. ${ }^{37}$ Philosophie wird bei Seneca wesentlich als Einübung in Verhaltensweisen und Einstellungen verstanden. Die literarische Form der Lucilius-Briefe darf demnach nicht als ein sekundäres Moment, als bloße "Verpackung" eines für sich vorhandenen philosophischen Gehalts vernachlässigt werden. Die beschriebene Form der Briefsammlung ist geeignet, den Übergang von der Explikation eines Begriffs der Philosophie zu einer Praxis des Philosophierens zu markieren und vorzubereiten.

37 Ep. 27,1: "Sic itaque me audi, tamquam mecum loquar: in secretum te meum admitto et te adhibito mecum exigo." - "So also höre mich an, als ob ich mit mir spräche: in meine Abgeschiedenheit lasse ich dich ein, und in deiner Gegenwart gehe ich streng mit mir zu Rate." Ep. 26,7: "Haec mecum loquor, sed tecum quoque me locutum puta."-»Das spreche ich mit mir, aber daß ich es auch mit dir gesprochen habe, stell dir vor." 